\title{
Automated Projection Spectroscopy (APSY) for the Assignment of NMR Resonances of Biological Macromolecules
}

\author{
Barbara Krähenbühl and Gerhard Wider*
}

\begin{abstract}
High resolution nuclear magnetic resonance (NMR) spectroscopy in solution is an established technique in structural biology. Detailed functional and structural studies of biological macromolecules by NMR require the assignment of the chemical shifts to specific nuclei. In biological applications, the necessary data is usually obtained from a number of two- and three-dimensional (2D and 3D) NMR experiments. Often, these data cannot be fully analyzed by automated computer programs due to insufficient separation and resolution of the signals in the available spectra. Then, complete resonance assignment requires manual interaction and can become a long and labor-intensive task. Automated projection spectroscopy (APSY) allows the substantial improvement of the resolution by providing spectral information from four and higher dimensional experiments without measuring the full spectrum, which would by far exceed any acceptable measuring time. APSY only measures a series of projections of the high-dimensional spectrum which can be obtained in a much shorter time. Peak picking of the projection spectra provides the basis for the calculation of the high-dimensional chemical shift correlation space by the algorithm GAPRO. The resulting high-dimensional peak lists are commonly artifact-free and of exceptional precision. Along with their high number of correlated nuclei they provide an ideal basis for reliable automated assignment. We will introduce the basic concepts of APSY, illustrate them with an application of a 6D APSY-seqHNCOCANH experiment, and discuss some practical aspects.
\end{abstract}

Keywords: APSY - Automated assignment - Automated projection spectroscopy •

High-dimensional experiments $\cdot$ Novel sampling approaches

\section{Introduction}

Nuclear magnetic resonance (NMR) in solution is a widely used tool in structural biology to provide data for studies of structural, functional and dynamic properties of biological macromolecules. ${ }^{[1]}$ The molecular weight of biological macromolecules studied with solution NMR ranges from approximately 3 to $100 \mathrm{kDa}(\mathrm{kg} / \mathrm{mol})$; the size of the investigated molecules is thus significantly larger than of those in usual chemistry applications. This results in several conceptual differences; especially the larger transverse relaxation rate and consequential line broadening leads to significantly lower sensitivity and resolution in NMR spectra of biological macromol-

\footnotetext{
${ }^{\star}$ Correspondence: Prof. Dr. G. Wider ETH Zurich

Institute of Molecular Biology \& Biophysics

Schafmattstrasse 30

$\mathrm{CH}-8093$ Zürich

Tel.: +4144633 3455

Fax: +41446331484

E-mail: gsw@mol.biol.ethz.ch
}

ecules. Broad signals and a much higher number of resonances make it impossible to separate individual resonance frequencies (chemical shifts) in ${ }^{1} \mathrm{H}$ NMR spectra, which are often sufficient for chemical applications. For improved separation 2D or 3D NMR spectra are measured. These multidimensional spectra often include frequencies of nuclei other than ${ }^{1} \mathrm{H}$ prevalent in biological macromolecules such as ${ }^{13} \mathrm{C}$ and ${ }^{15} \mathrm{~N}$ for proteins and in addition ${ }^{31} \mathrm{P}$ for RNA and DNA. Due to their low natural abundance ${ }^{13} \mathrm{C}$ and ${ }^{15} \mathrm{~N}$ isotopes must be enriched to $>98 \%$ upon production of the macromolecules for sufficient sensitivity of the spectra.

A detailed analysis of NMR spectra requires the assignment of the chemical shifts to the individual nuclei. This assignment procedure is usually based on multidimensional spectra that use the bondingelectron-mediated isotropic (scalar) coupling to correlate covalently bound nuclei. With a suitable radio-frequency pulsing scheme (pulse sequence) magnetization (coherence) is transferred via the throughbond interactions along the nuclear pathway of interest to a final nucleus, usually a proton, on which the signal is detected as free induction decay (FID). The chemi- cal shift information of a nucleus along the pathway can be included by repeating the transfer many times and at the same time incrementing the appropriate evolution period in the pulse sequence. The chemical shifts manifested during the evolution periods are indirectly detected through amplitude-modulation of the signals contained in the FID. Fourier transformation in the acquisition dimension and subsequently in the indirect dimensions leads to the multidimensional correlation spectrum.

Conventional NMR experiments are limited to two or three correlation dimensions even though they may include more nuclei along the coherence transfer pathway. For higher dimensional experiments unacceptable acquisition times are obtained since the overall measurement time scales with the product of the number of points in the indirect dimensions. Whereas a 3D experiment takes one to three days, a 4D experiment can easily take weeks to months, and a 5D or even higher-dimensional experiment would take years. Even 3D experiments are often acquired with reduced resolution in the indirect dimensions to stay within acceptable measurement times. Impaired resolution and limited dimensionality leads to signal 


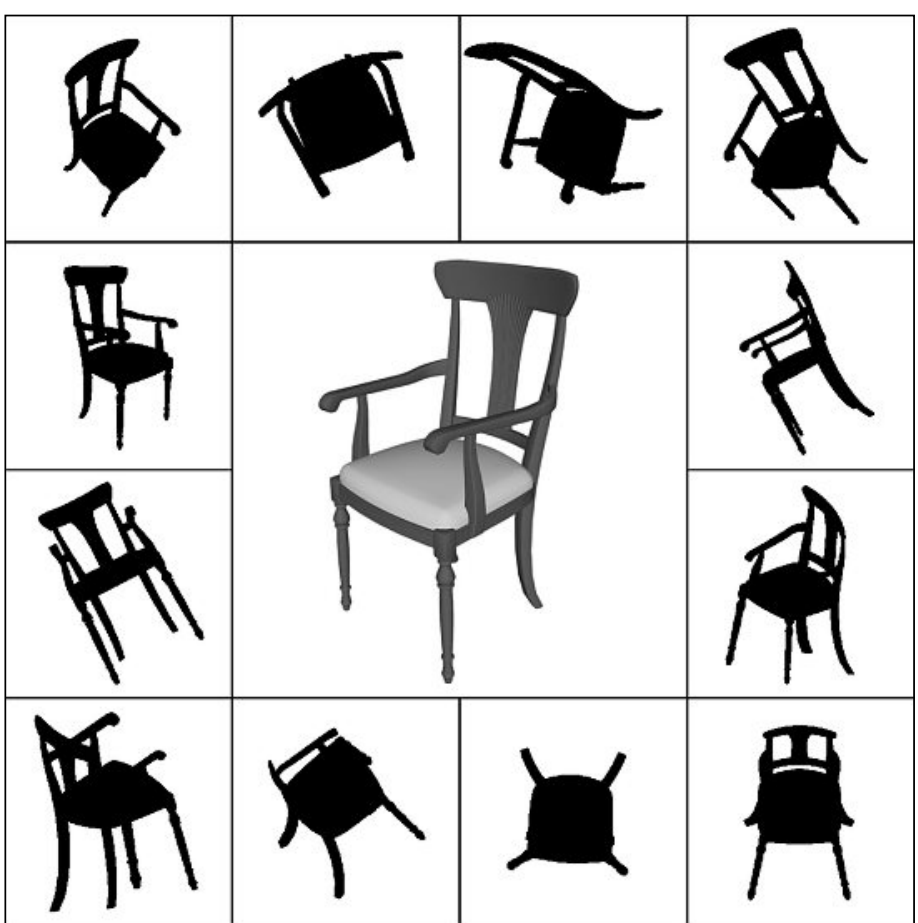

Fig. 1. Visualization of the frequently used image-based 3D reconstruction method as an illustration for the underlying concept of automated projection spectroscopy (APSY). A set of images (corresponding to $2 \mathrm{D}$ projections) of the object are taken from different angles. For a grid of characteristic points their coordinates in 3D space are calculated based on their varying position in the different projections; the resulting grid serves as a basis to create a spatial 3D model of the object. For a faithfu $3 \mathrm{D}$ reconstruction further steps such as texture reconstruction would be necessary. However, for APSY the crucial information, the multidimensional coordinates (chemical shifts), is available at this point.

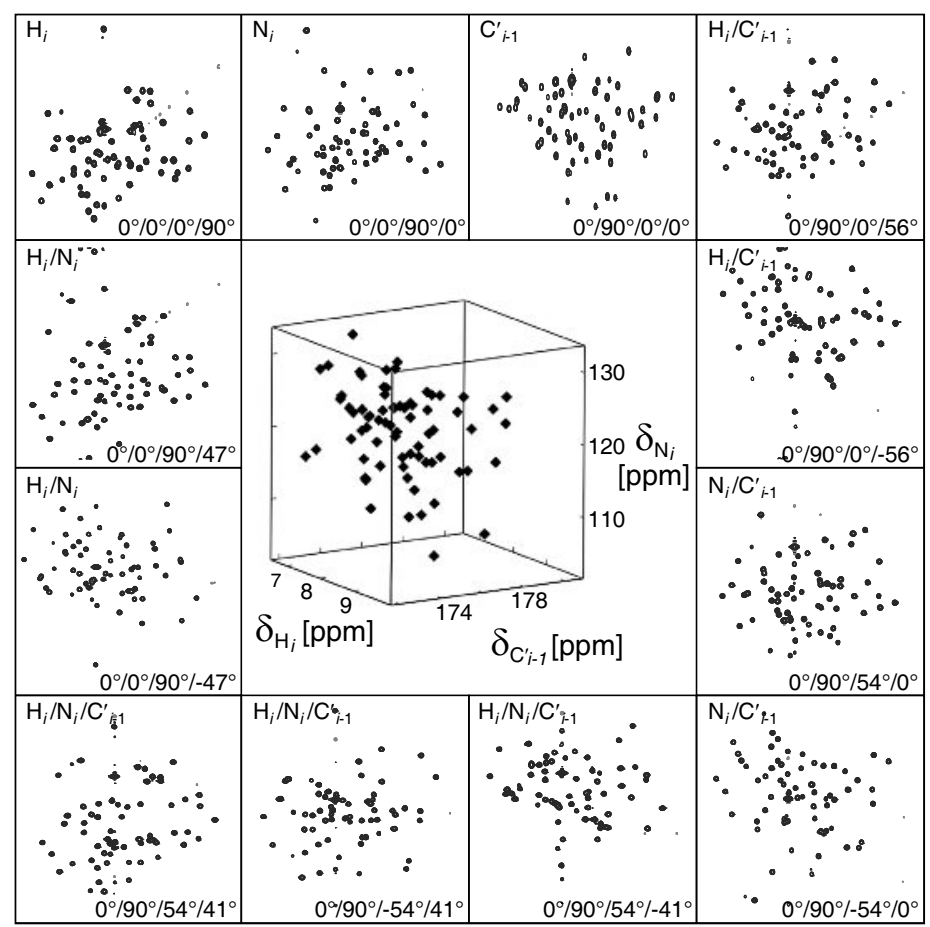

Fig. 2. Illustration of the principle of automated projection spectroscopy (APSY) with a selection of 12 2D projections from a 6D APSY-seqHNCOCANH experiment measured on the protein ubiquitin. This figure includes a subset of all 2D projection spectra; only projections with evolution periods for the amide group nuclei $\mathrm{H}_{i}, \mathrm{~N}_{i}$ and $\mathrm{C}^{\prime}{ }_{i-1}$, are shown. The nuclei contained in the indirect dimensions are indicated in the top left corner of the individual projections. In the cube shown in the center their calculated 3D peak positions are illustrated representative for the hardly visualizable full $6 \mathrm{D}$ space. The values specified at the bottom right of each spectrum are the projection angles $\alpha, \beta, \gamma$ and $\delta$. The horizontal axis of the projection spectra represents the acquisition dimension with the chemical shifts of $\mathrm{H}_{i-1}$. In the indirect dimension (vertical axis) the positions of the 2D cross peaks are angle-dependent superpositions of the chemical shifts of the nuclei indicated in the individual projections. overlap, which impedes automated assignment strategies. Thus, numerous 2D and $3 \mathrm{D}$ correlation spectra are required and manually supported analysis becomes necessary, which is not only a labor-intensive task but can also lead to biased results. New acquisition schemes enable acquisition of high-dimensionality experiments ${ }^{[2]}$ within an acceptable time. One promising approach is automated projection spectroscopy (APSY) ${ }^{[2 \mathrm{f}]}$ which allows experiments to be measured with high dimensionality ( $\geq 3$ ) and optimal spectral resolution.

APSY is based on projection spectroscopy which descends from reduced dimensionality experiments. ${ }^{[2]}$ The latter are based on the simultaneous (instead of sequential) incrementation of several evolution periods from more than one indirect dimension. Separation of individual components of the signals obtained with this sampling pattern leads to spectra which correspond to projections of the high dimensionality signal space - the concept of projection spectroscopy. ${ }^{[3]}$ From a series of projections the full spectrum can be reconstructed. ${ }^{[3 a, c]}$ There are a number of reconstruction algorithms; however, all of them introduce some artifacts which reduce spectral quality and make the necessary peak peaking difficult. APSY circumvents these problems since it does not use reconstruction. For APSY a series of projections (usually $2 \mathrm{D}$ projections) of an NMR experiment with typically 4 to 7 dimensions is measured, processed, and peak picked. The resulting chemical shift correlation lists (peak lists) of the projections are then used to calculate the full high-dimensional peak list with the algorithm GAPRO.[2f] This algorithm also filters artifacts which potentially appear in any NMR spectrum, and averages the chemical shift information from the already quite well-resolved 2D projections, leading to accurate and artifact-free peak lists with highly precise chemical shift values. An adequate set of these peak lists provides a reliable basis for subsequent automated assignment strategies.

The basic idea of APSY is illustrated in Fig. 1. Based on a series of pictures (2D projections) of an object a model in 3D space can be calculated. From a network of characteristic points identified in the images their exact position in 3D space can be calculated by analyzing their relative position in different images taken from different angles - a similar procedure is applied by the algorithm GAPRO. Image-based reconstruction would proceed with more details, such as e.g. the texture, but for APSY the required information is already attained at this point: the key information is not the spectrum but the chemical shifts in the multidimensional space. The approach to select the essential information (chemical shifts) already in the projection spectra allows obtaining the pure high dimensionality peak position information fast and in high quality. In the following section we will outline the APSY concept; a more detailed account can be found in a recent review. ${ }^{[4]}$

\section{The Basic Concepts of APSY}

Automated projection spectroscopy (APSY) makes the information content of $n$-dimensional $(n \mathrm{D}) \mathrm{NMR}$ experiments available by analyzing a series of $2 \mathrm{D}$ projections of the $n \mathrm{D}$ spectrum at different projection angles (Fig. 1 and 2). The 
value of the dimensionality $n$ must be at least 3; 4D and 5D APSY experiments are the most common ones, the APSY experiment with the highest dimensionality so far has seven dimensions; relaxation imposes limits to the dimensionality. The experiments used in APSY are often similar to corresponding conventional experiments, e.g. employing the same magnetization transfer pathways. The main differences in the pulse sequences are modifications to measure the resonance frequencies for all nuclei along the pathway. Thus, APSY experiments usually contain additional evolution periods, i.e. more indirect dimensions resulting in higher dimensionality of the experiment. Since the method allows for longer evolution periods and therewith better resolved spectra the pulse sequence is designed to minimize the influence of scalar coupling and relaxation whenever feasible. The acquisition of $2 \mathrm{D}$ projections of the $n \mathrm{D}$ signal space is accomplished by measuring data points on a cross section in the time domain, as is illustrated with dots in Fig. 3. The projection-cross section theorem states that a cross section in the time domain corresponds after Fourier transformation to a projection at the same angle in the frequency domain. ${ }^{[5]}$ In practice, the simultaneous incrementation of two different evolution periods leads to a modulation of the chemical shifts with each other resulting in a splitting of the signal into two. They correspond to the sum of the projections with angles $\pm \alpha$ which are not separated. For the separation into single projections with angle $+\alpha$ and $-\alpha$ a second spectrum recorded with the same projection angle is required, but with a change in the pulse sequence analogous to quadrature detection (i.e. distinction of positive and negative frequencies) in indirect dimensions of multidimensional experiments. ${ }^{[3 c, 6]}$ In general, $m$ projected dimensions result in a splitting into $2^{(m-1)} \mathrm{sig}$ nals which correspond to $2^{(m-1)}$ projections along $2^{(m-1)}$ angle sets each with the same $(m-1)$ non-orthogonal projection angles. A set of $2^{(m-1)}$ spectra is measured in a way that the separate projection spectra can be extracted upon proper addition/subtraction of these measured spectra, as is described by Hiller et al. ${ }^{[2 \mathrm{f}]}$ From the set of 2D projections of the $n \mathrm{D}$ experiment APSY does not reconstruct the original spectrum (or chair in Fig. 1), but calculates only the resonance positions (chemical shifts) in the $n \mathrm{D}$ experiment (Fig. 2), thus the result of APSY will be a list of the chemical shifts of the peaks that are present in the $n \mathrm{D}$ spectrum.

In the APSY procedure the information content of the set of $2 \mathrm{D}$ projection spectra is extracted by a simple peak picking algorithm. ${ }^{[7]}$ From $k$ projection spectra $k$ peak lists are obtained which are passed to the algorithm GAPRO.[2f] This algorithm

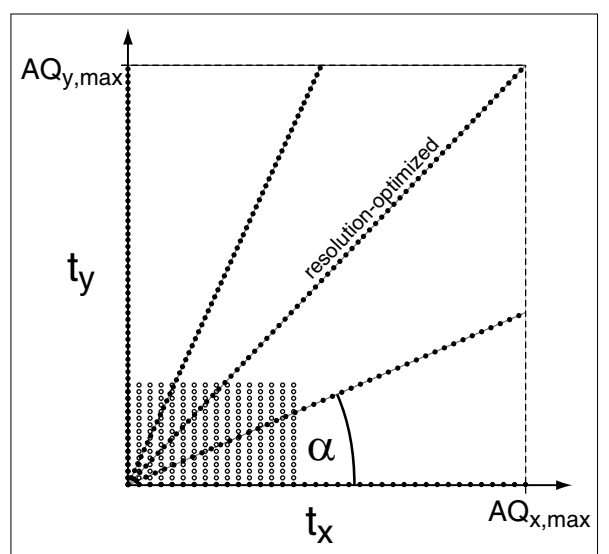

Fig. 3. The sampling patterns in the time domain with two indirect dimensions are illustrated by black dots for five projections as used in automated projection spectroscopy (APSY). The rectangular area filled with circles represents a conventional sampling scheme for the indirect dimensions of a 3D experiment. The axis labeled with $t_{x}$ and $t_{y}$ are the evolution periods of nuclei $x$ and $y . A Q_{x, \max }$ and $A Q_{y, \max }$ are the maximal evolution periods in the respective dimension. The sampling cross section for the projection labeled with 'resolution-optimized' represents the projection for which both evolution periods can be fully exploited.

forms the 'heart' of APSY, and accounts for most of its features and advantages. From a subset of the peak lists of the projections GAPRO calculates possible peak positions, candidate peaks, in the $n \mathrm{D}$ spectrum and evaluates them by counting the number of $2 \mathrm{D}$ peak lists in which the projection of the $n \mathrm{D}$ peak appears - the support value. The candidate point with the highest support value (or a random choice of one when equal support values exist) is included in an $n \mathrm{D}$ peak list and removed. The support of any other candidate point is reduced by the number of projections where it coincides with the removed peak. The procedure is repeated until the support values reach a user-defined threshold value, which represents the minimal number of projections in which each final multidimensional peak should appear. At this point these iteration steps are repeated with a new random subset of projection peak lists. After the user-defined repetition value, the results from the different runs are averaged leading to highly accurate and precise $n \mathrm{D}$ chemical shift values due to statistical selection and averaging effects. The precision of an APSY peak list lies usually beyond any feasible experimental spectral resolution, since the chemical shifts are averages from a series of peak positions in the projection spectra. The final chemical shift precision depends on the signal separation, on the number of measured projections and on the resolution in the projection spectra. Precision improvements of a factor of 10 to 100 with respect to conventionally deter- mined chemical shift values are common when using APSY.

For projection spectra the choice of projection angles is an important criterion for optimal separation of signals. There are two main factors to be considered when creating a suitable set of projection angles: resolution and dispersion. For APSY usually $2 \mathrm{D}$ projections are measured for which the number of points measured in the indirect dimension can be set to the experimentally optimal value to optimize resolution, in contrast to conventional 3D or even higher-dimensional spectra. There is one angle set for each combination of indirect dimension nuclei which allows maximal resolution for all included nuclei, such as the cross section in Fig. 3 which is indicated with 'resolution-optimized'. The maximal experimentally possible resolution is determined by constant-time evolution limits, or coupling or relaxation induced signal modulations. Dispersion optimization in APSY aims at balancing the distinct chemical shift ranges of different nuclei, as is explained in detail by Hiller et al. ${ }^{[7]}$ Another aspect for the selection of angle sets arises from the effect that the sensitivity of any measured spectrum is reduced by a factor of $\sqrt{ } 2$ per additional indirect dimension. In APSY this effect is minimized by measuring not more than three simultaneously incremented evolutions per projections, i.e. not more than two non-orthogonal projection angles are used.

At present there is a wide variety of published and established APSY-type experiments for protein backbone and side chain assignment. ${ }^{[8]}$ For nucleic acids a pivotal experiment has been published: a 5D APSY-HCNCH sugar-to-base correlation experiment. ${ }^{[9]}$ Further experiments for the assignment of large proteins and nucleic acids are currently being finalized. An application with a 6D APSY-seqHNCOCANH experiment ${ }^{[8 a]}$ is presented in the next section.

\section{Sequential Protein Backbone Assignment with a 6D APSY-seq- HNCOCANH Experiment}

In order to clarify the APSY concepts we illustrate them with a 6D APSY-seqHNCOCANH experiment. ${ }^{[8]}$ This experiment results in a $6 \mathrm{D}$ peak list which can directly be used for sequence-specific protein backbone assignment. The pulse sequence of the experiment transfers the magnetization through covalent bonds starting at the protons of the backbone amide groups ${ }^{1} \mathrm{H}_{i}$ and proceeding via ${ }^{15} \mathrm{~N}_{i},{ }^{13} \mathrm{C}^{\prime}{ }_{i-1},{ }^{13} \mathrm{C}^{\alpha}{ }_{i-1}$ and ${ }^{15} \mathrm{~N}_{i-1}$ to ${ }^{1} \mathrm{H}_{i-1}$, where the signal is detected. The indices $i$ and $i-1$ represent the relative residue number counted from the amino acid where the transfer starts. The 
chemical shift of each nuclei on the coherence transfer pathway (Fig. 4) is measured with an evolution period. All five evolution periods use time periods which are necessary for the magnetization transfer also in conventional versions of the experiment. However, the ${ }^{1} \mathrm{H}_{i}$ and ${ }^{13} \mathrm{C}^{\prime}{ }_{i-1}$ evolution periods require some additional time to obtain sufficient resolution. The combination of transfer and evolution time periods makes the cost in sensitivity of the 6D APSY version compared to conventional versions rather low.

The 6D APSY-seq-HNCOCANH experiment was measured with the protein ubiquitin, which has 76 amino acids including three prolines whose amide is tertiary and therefore not covered with this experiment. 33 projections were acquired within 6 hours; some of these projection spectra are presented in Fig. 2. The measurements were performed with a $1.9 \mathrm{mM}$ sample at $25{ }^{\circ} \mathrm{C}$ on a Bruker $500 \mathrm{MHz}$ (11.7 Tesla) NMR spectrometer equipped with a cryogenically cooled probe and operated by the software Topspin 3.0 (Bruker, Karlsruhe, Germany). Spectral sweep widths of 1900 $\mathrm{Hz}, 1723 \mathrm{~Hz}, 1509 \mathrm{~Hz}, 3521 \mathrm{~Hz}, 1723 \mathrm{~Hz}$ and $7002 \mathrm{~Hz}$ were set for ${ }^{1} \mathrm{H}_{\mathrm{i}},{ }^{15} \mathrm{~N}_{i},{ }^{13} \mathrm{C}^{\prime}{ }_{i-1}$, ${ }^{13} \mathrm{C}^{\alpha}{ }_{i-1},{ }^{15} \mathrm{~N}_{i-1}$ and the acquisition dimension ${ }^{1} \mathrm{H}_{i-1}{ }^{i-1}$, respectively, and maximal evolution times of $30 \mathrm{~ms}, 28 \mathrm{~ms}, 20 \mathrm{~ms}, 20 \mathrm{~ms}, 28 \mathrm{~ms}$ and $73 \mathrm{~ms}$ were used. The experimental parameters were entered in one $6 \mathrm{D}$ parent data set from which automatically an evolutionoptimized angle set for the projections was calculated and the corresponding experiment data sets were created. The measured data was serially processed, in our case with the software PROSA, ${ }^{[10]}$ but any other processing software can be used as well. Peak picking in the 33 projection spectra and the subsequent calculation of the $6 \mathrm{D}$ HNCOCANH peak list was accomplished by GAPRO resulting in 72 cross peaks. With the assignment software MARS $1.2^{[11]} 100 \%$ of the resonances which are expected for ubiquitin with this experiment were sequence-specifically assigned to the backbone atoms of ubiquitin. All 6D correlations which would start or end on a proline residue or on the N-terminal amino acid are naturally not appearing in the $6 \mathrm{D}$ peak list; for ubiquitin correlations with glycine 53 are not detected, a residue which is usually missing already in conventional $\left[{ }^{15} \mathrm{~N},{ }^{1} \mathrm{H}\right]-\mathrm{HSQC}$ experiments. ${ }^{[12]}$ For ubiquitin a maximum of $92 \%$ of all backbone resonances can be assigned with a 6D HNCOCANH experiment, which we achieved. The computational time required for all processing steps including GAPRO and MARS analysis was less than $5 \mathrm{~min}$ on a standard computer work-station. Including some interactive steps such as copying data, starting software, evaluating the data and optimizing parameters, the

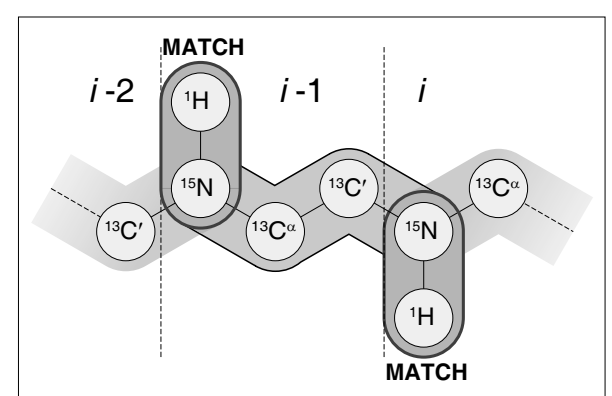

Fig. 4. Schematic representation of the mag netization transfer pathway and matching procedure for the 6D APSY-seq-HNCOCANH experiment. ${ }^{[8 \mathrm{a}]}$ The experiment is intended for sequence specific backbone resonance assignment of proteins. The relative position of the amino acid in the protein sequence is labeled with $i, i-1$ and $i-2$, with $i$ denoting the amino acid where the magnetization transfer starts. The chemical shifts in the 6D peak list resulting from this experiment are matched via the ${ }^{1} \mathrm{H}$ and ${ }^{15} \mathrm{~N}$ values and connected to fragments which can be sequence-specifically assigned to the protein backbone. The matching procedure is illustrated with the bold line around the ${ }^{1} \mathrm{H}-{ }^{15} \mathrm{~N}$ group and the word MATCH.

total time required for the backbone resonance assignment was about 1 hour once data accumulation was finished.

The 6D APSY compares favorably to conventional $2 \mathrm{D}$ and $3 \mathrm{D}$ experiments using the same HNCOCANH coherence transfer pathway. For example a $3 \mathrm{D} \mathrm{H}_{i-1}$ $\mathrm{N}_{i-1}-\mathrm{N}_{i}$ experiment with the same maximal number of points in the indirect dimensions as the 6D would take approximately 21 hours, more than three times as long as the full 6D APSY-seq-HNCOCANH. In the assignment procedure the ${ }^{15} \mathrm{~N}$ chemical shifts in the 3D spectrum must be matched. Since the spectral resolution in the ${ }^{15} \mathrm{~N}$ dimensions is at best about $20 \mathrm{~Hz}(0.4 \mathrm{ppm}$ at $500 \mathrm{MHz}$ ) in many cases manual interaction will be required, and even this may fail in some cases of resonance overlap. In contrast, APSY profits from an optimal separation of the signals in six dimensions and provides with ${ }^{1} \mathrm{H}$ and ${ }^{15} \mathrm{~N}$ in the amide group two chemical shifts for matching compared to only one in the 3D experiment. Further, APSY results in an average precision of $0.004 \mathrm{ppm}$ and $0.0004 \mathrm{ppm}$ in the ${ }^{15} \mathrm{~N}$ and ${ }^{1} \mathrm{H}$ dimension, respectively, determined as average chemical shift deviation for the same backbone ${ }^{1} \mathrm{H}-{ }^{15} \mathrm{~N}$ group measured in different dimensions.

\section{Discussion}

APSY provides a method which takes advantage of the resolution of high-dimensional spectra. Only low-dimensional projection experiments (mostly only 2D) are measured, and based on peak positions in these projections the $n \mathrm{D}$ peak list is calculated without reconstruction of the $n \mathrm{D}$ spectrum. These $n \mathrm{D}$ peak lists offer exceptionally high chemical shift precision and provide an ideal basis for subsequent automated resonances assignments. The high precision of APSY peak lists was independently confirmed by an application with an unfolded protein with 441 amino acids ${ }^{[13]}$ which was sequence-specifically assigned using APSY. Due to resonance overlap and pure resolution this assignment could not have been obtained based on conventional NMR spectra.

For a successful and efficient application of APSY, e.g. for the assignment of a protein, some points must be considered. Most criteria for a sample to be suitable for APSY are identical to conventional applications of biological NMR methods. The sample to be investigated must be a highly pure solution of the molecule of interest with a concentration of more than $500 \mu \mathrm{M}$ in a volume $>280 \mu$ l. Salts can reduce the NMR sensitivity severely and should not exceed a concentration of $\sim 100 \mathrm{mM}$. Most high-dimensionality experiments with proteins and RNA/DNA require isotope-labeling with ${ }^{15} \mathrm{~N}$ and ${ }^{13} \mathrm{C}$. A further important point is the stability of the molecule: any change in the sample such as degradation during the measurements can impede the quality of the spectra and consequentially impair an automated assignment procedure. The magnetization transfer pathways in high-dimensional NMR experiments can take a substantial time, and transverse relaxation may reduce the sensitivity. For example for a folded, globular protein relaxation will limit the application of APSY to a maximal size of 15 to $20 \mathrm{kDa}$, whereas for an unfolded protein the size can be as large as $50 \mathrm{kDa} .^{[13]}$ When the protein is deuterated the molecular weight range for a folded protein can be extended to about $25 \mathrm{kDa}$, and even more when special transverse relaxation optimized techniques (TROSY) ${ }^{[14]}$ are used. The usefulness of a particular APSY experiment for the molecule of interest can be checked by measuring the $2 \mathrm{D}$ projections of the multidimensional experiments with only one type of nucleus in the indirect dimension. These are the orthogonal projections, such as the tree projections on the top left of Fig. 2; their sampling pattern is indicated in Fig. 3 along the axes. Comparison with conventional 2D heteronuclear correlation experiments allows estimating the sensitivity and completeness of the high dimensionality APSY spectra.

A usual set of APSY experiments for automated assignment on proteins consists of 4D and 5D experiments, e.g. of a 4D APSYHACANH, a 5D APSY-HACACONH and a 5D APSY-CBCACONH experiment. [7] The use of higher dimensional experi- 
ments such as the discussed 6D APSY-seqHNCACONH experiment or the 7D APSY$\mathrm{HC}(\mathrm{CC}-\mathrm{TOCSY}) \mathrm{CONH}$ experiment ${ }^{[8 \mathrm{~b}]}$ is limited to samples with strong signals such as unfolded (non-globular), small and/or highly concentrated proteins. The pulse programs of the experiments, the projection setup script for Bruker spectrometer, and the software GAPRO are provided online (www.apsy.ch). The assignment procedure (matching) can performed by alternative software to MARS, ${ }^{[11]}$ e.g. MATCH, ${ }^{[15]}$ GARANT ${ }^{[16]}$ or FLYA. ${ }^{[17]}$

The method is being further developed in our group for applications on proteins and nucleic acids. The APSY principle, however, might be useful also for other applications of NMR beyond our scope. Any high-dimensionality experiment can profit from the concept. For 3D experiments we recommend it for cases where exceptionally high precision of the final chemical shift values are required, since APSY allows the precision to be controlled with the number of measured projections.

\section{Acknowledgements}

We thank Prof. Sebastian Hiller (University Basel) for technical discussions. The Swiss National Fund (SNF) is gratefully acknowledged for financial support (project 200021_120048).

Received: August 2, 2012

[1] K. Wüthrich, Angew. Chem. Int. Ed. 2003, 42, 3340.

[2] a) T. Szyperski, G. Wider, J. H. Bushweller, K. Wüthrich, J. Am. Chem. Soc. 1993, 115, 9307; b) P. Schmeider, A. S. Stern, G. Wagner, J. C. Hoch, J. Biomol. NMR 1994, 4, 483; c) K. Aggarwal, M. A. Delsuc, Magn. Reson. Chem. 1997, 35, 593; d) V. Y. Orekhov, I. V. Ibraghimov, M. Billeter, J. Biomol. NMR 2001, 20, 49; e) E. Kupce, T. Nishida, R. Freeman, Prog. Nucl. Magn. Reson. Spectrosc. 2003, 42, 95; f) S. Hiller, F. Fiorito, K. Wüthrich, G. Wider, Proc. Natl. Acad. Sci. USA 2005, 102, 10876; g) B. E. Coggins, P. Zhou, J. Magn. Reson. 2006, 182, 84; h) K. Kazimierczuk, A. Zawadzka, W. Kozminski, I. Zhukov, J. Biomol. NMR 2006, 36, 157.

[3] a) E. Kupce, R. Freeman, J. Am. Chem. Soc. 2003, 125, 13958; b) S. Kim, T. Szyperski, J. Am. Chem. Soc. 2003, 125, 1385; c) E. Kupce, R. Freeman, J. Am. Chem. Soc. 2004, 126, 6429.

[4] S. Hiller, G. Wider in 'Automated Projection Spectroscopy and Its Applications', Vol. 316, Eds. M. Billeter, V. Orekhov, Springer Berlin Heidelberg, 2011, p. 21.
[5] a) K. Nagayama, P. Bachmann, K. Wüthrich, R. R. Ernst, J. Magn. Reson. 1978, 31, 133; b) R. M. Mersereau, A. V. Oppenheim, P Ieee 1974, 62, 1319.

[6] B. Brutscher, N. Morelle, F. Cordier, D. Marion, J. Magn. Reson., Ser B 1995, 109, 238.

[7] S. Hiller, G. Wider, K. Wüthrich, J. Biomol. NMR 2008, 42, 179.

[8] a) F. Fiorito, S. Hiller, G. Wider, K. Wüthrich, J. Biomol. NMR 2006, 35, 27; b) S. Hiller, C. Wasmer, G. Wider, K. Wüthrich, J. Am. Chem. Soc. 2007, 129, 10823; c) S. Hiller, R. Joss, G. Wider, J. Am. Chem. Soc. 2008, 130, 12073; d) A. D. Gossert, S. Hiller, C. Fernandez, J. Am. Chem. Soc. 2011, 133, 210; e) B. Krähenbühl, S. Hiller, G. Wider, J. Biomol. NMR 2011, 51, 313.

[9] B. Krähenbühl, D. Hofmann, C. Maris, G. Wider, J. Biomol. NMR 2012, 52, 141.

[10] P. Güntert, V. Dötsch, G. Wider, K. Wüthrich, J. Biomol. NMR 1992, 2, 619.

[11] Y. S. Jung, M. Zweckstetter, J. Biomol. NMR 2004, 30, 11 .

[12] N. Tjandra, S. Grzesiek, A. Bax, J. Am. Chem. Soc. 1996, 118, 6264

[13] R. L. Narayanan, U. H. N. Dürr, S. Bibow, J. Biernat, E. Mandelkow, M. Zweckstetter, J. Am. Chem. Soc. 2010, 132, 11906.

[14] K. Pervushin, R. Riek, G. Wider, K. Wüthrich, Proc. Natl. Acad. Sci. USA 1997, 94, 12366.

[15] J. Volk, T. Herrmann, K. Wüthrich, J. Biomol. NMR 2008, 41, 127.

[16] C. Bartels, P. Guntert, M. Billeter, K. Wüthrich, J. Comput. Chem. 1997, 18, 139.

[17] E. Schmidt, P. Güntert, J. Am. Chem. Soc. 2012, 134, 12817. 